\title{
Preliminary Results from DØ Run II Data
}

\author{
Wagner de Paula Carvalho (for the DØ Collaboration) \\ Instituto de Física, Universidade Estadual do Rio de Janeiro, \\ Rua São Francisco Xavier 524, 20550-900, Rio de Janeiro, RJ, Brazil
}

Received on 01 August, 2003.

\begin{abstract}
We present recent physics results from the data collected by the DØ detector in this early stage of Run II of the Tevatron proton-antiproton collider. Emphasis is given to the Forward Proton Detector, a new subsystem that will enable detailed studies of diffractive phenomena at Tevatron.
\end{abstract}

\section{Introduction}

The first period of running of the Fermilab Tevatron (called Run I), lasting from 1992 to 1996, was very successful in producing high quality physics results.

The DØ Collaboration [1], one of the two broad international collaborations operating in the collider ring (The other one being the CDF - Collider Detector at Fermilab), had a extensive and well succeed physics program that gave rise to more than one hundred physics papers published in world class publications. Topping its achievements is top quark discovery (announced at the same time by CDF), but a extensive list of $\mathrm{D} \varnothing$ Run I physics highlights can be found in [2].

The ongoing Run II of the Tevatron was planned to significantly extend this physics program. The accelerators and detectors upgrades should allow the two collaborations to greatly improve over and refine many of the measurements performed with Run I data. New measurements shall also be made possible and, perhaps, some new physics become accessible.

The DØ Run II menu includes topics like: make precision measurements of the top quark properties; $t \bar{t}$ spectroscopy as sign of new physics; refine measurements on the electroweak sector, especially gauge boson couplings and $W$ mass; mixing, $\mathrm{CP}$ violation and $b$ hadron spectroscopy in the $b$ sector, as well as extend $b$-quark production measurements to new kinematic regions; search for new phenomena (leptoquarks, SUSY, technicolor, etc.); extend the jet measurements to further constrain proton structure functions; study a variety of diffractive phenomena (hard diffraction, double diffraction, double pomeron exchange, diffractive heavy flavor production); search for glueball states.

\section{The Upgraded Tevatron}

The Fermilab accelerator complex has gone through a major upgrade from Run I to Run II. The Antiproton Source and the Booster were improved and the old Main Ring, that shared the same tunnel with the Tevatron, was shut down and replaced by the Main Injector.

Such improvements were aimed to increase Tevatron energy and luminosity. The center of mass energy went up slightly from 1.80 to $1.96 \mathrm{GeV}$, which is enough nevertheless to increase $t \bar{t}$ production cross section by nearly $40 \%$. The beam bunch structure has changed, going from 6 bunches of protons $\times 6$ bunches of antiprotons to $36 \times 36$. The time interval between successive bunch crossings has shortened from $3.5 \mu$ s to $396 \mathrm{~ns}$ and the instantaneous luminosity is expected to increase from typically $1.6 \times 10^{30} \mathrm{~cm}^{-2} \mathrm{~s}^{-1}$ to a projected value of $8.6 \times 10^{31} \mathrm{~cm}^{-2} \mathrm{~s}^{-1}$.

The integrated luminosity during the whole Run I was a little above $100 \mathrm{pb}^{-1}$. With all these accelerator changes, the expected accumulated luminosity for the first phase of the Run II (Run IIa), ending in 2004, is $2 \mathrm{fb}^{-1}$. The accelerator will then be subject to new improvements and the total Run II integrated luminosity should reach $15 \mathrm{fb}^{-1}$.

\section{The Upgraded DØ Detector}

The DØ Detector [3] was also subject of a major upgrade from Run I to Run II [4].

The old central detector comprised of drift chambers and a transition radiation detector was replaced by silicon microstrip detectors (SMT) and a fine segmented scintillating fiber system (CFT), both placed inside a $2 \mathrm{~T}$ superconducting solenoid (there was no central magnetic field in the $\mathrm{D} \varnothing$ Run I Detector).

Preshower detectors were installed before the calorimeters to aid electron identification and triggering and to correct electromagnetic energy for effects of the solenoid.

The muon system in the forward region $(|y|>1)$ [5] was completely reformulated. In Run I there was an intermediate system $(1.0<|y|<1.6)$ and a small angle system $(2.4<|y|<3.2)$, each one using a different type of proportional drift tubes. They were replaced by a new system based on mini drift tubes covering the rapidity region $1<|y|<2$. Also new layers of scintillators for triggering and background rejecting purposes were added to both, the central $(|y|<0.8)$ and forward region. 
Another major change was the addition of a set of forward proton and antiproton detectors. Placed inside Roman pots, ranging from $23 \mathrm{~m}$ to $59 \mathrm{~m}$ away from the $\mathrm{D} \varnothing$ center, they added to the $\mathrm{D} \varnothing$ spectrometer the capability of tagging scattered protons and antiprotons.

Finally, entirely new trigger and DAQ systems had to be designed and installed to cope with the increased event rate of Run II.

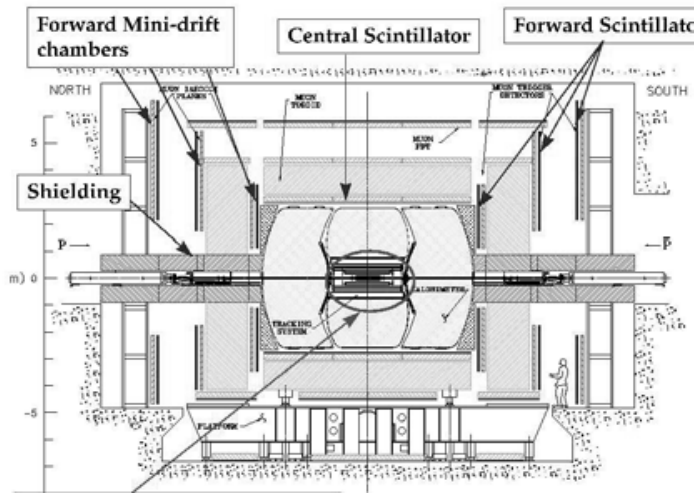

Figure 1. Schematic side view of the $\mathrm{D} \emptyset$ upgraded detector.

\section{Detector Performance}

After just a few months of stable operation of most of the detector systems, they seem to be producing good quality data.

The new central detector is having a good performance, approaching some of the designed goals. SMT and CFT detection efficiencies are above $97 \%$ and $98 \%$, respectively. The impact parameter resolution for tracks reconstructed in the central detector is shown in Fig. 2 and compared to the expected parameterization. One can see a reasonable agreement, with only survey alignment taken into account. Including alignment with data should improve the reconstruction.

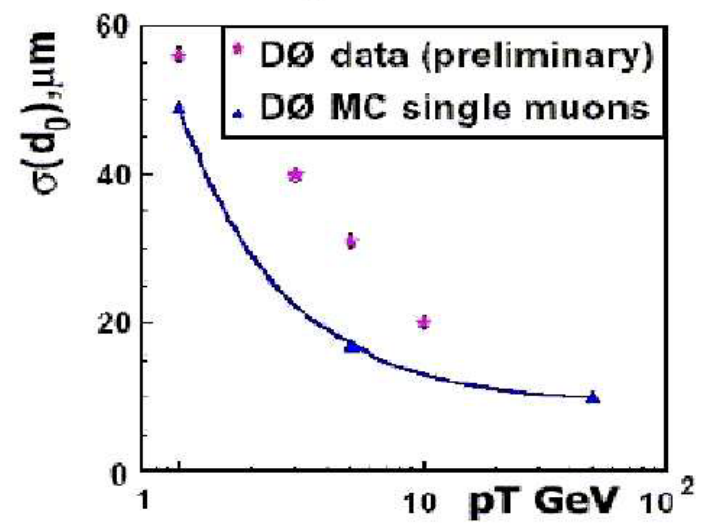

Figure 2. Measured vs. predicted impact parameter for single muons.
The muon system also seems to be functioning properly and the scintillators time resolution of $2.5 \mathrm{~ns}$ is proving helpful in obtaining clean muon samples by removing the outof-time background.

The calorimeters preserve Run I excellent performance and good energy resolution, with only about $0.1 \%$ dead channels out of their $55 \mathrm{~K}$ readout channels.

\section{Physics Results}

The Run II started in March, 2001. About $50 \mathrm{pb}^{-1}$ have been delivered so far, with peak instantaneous luminosity reaching $33.5 \times 10^{30} \mathrm{~cm}^{-2} \mathrm{~s}^{-1}$. Most of this luminosity was used for detectors commissioning. The results shown next are based on approximately $10 \mathrm{pb}^{-1}$ of physics quality data.

\subsection{B-Physics}

The first results on B-Physics are related to the production of the $b$ quark.

So far, $b$-quark production at $\mathrm{D} \varnothing$ has been tagged by the presence of muons in the event, which is a characteristic signature of B hadron direct or indirect decay.

Muons are triggered and identified through the signals they leave in the three layers of proportional drift chambers and in the scintillators (2 layers in the central and 3 layers in the forward region). A fit to the reconstructed track segments before and after the magnetized iron toroid gives a measure of the muon momentum.

Due to the large material amount before the muon chambers, $\mathrm{D} \varnothing$ has the capability of detecting muons inside jets. Fig. 3 shows a preliminary Run II $\mu+$ jet cross section as a function of the jet $E_{T}$ for muons of $p_{T}>4 \mathrm{GeV} / \mathrm{c}$ detected in the central muon system. The jet algorithm used a cone size $\mathcal{R} \equiv \sqrt{\Delta \eta^{2}+\Delta \phi^{2}}=0.5$.

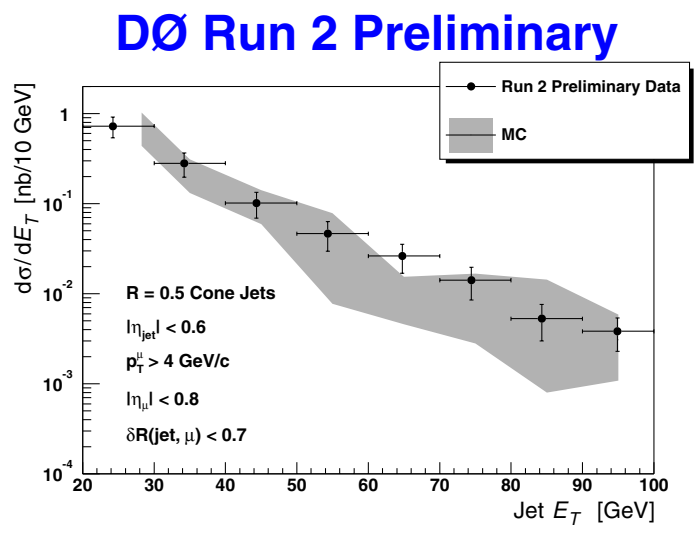

Figure 3. Muon + jet cross section.

This is the first step toward the determination of the $b$ jet cross section. The fraction of these muons which comes 
from $b$-quarks can be inferred by fitting the transverse momentum of the muon with respect to the jet axis $\left(p_{T}^{r e l}\right)$.The estimated $b$ content in this sample is $21.5 \pm 4.3 \%$.

$J / \Psi$ decaying in two muons is an important signature of $b$-quark production at hadron colliders. Clean signals may be obtained and used to extract inclusive production rates or select exclusive samples. Fig. 4 shows preliminary results on $J / \Psi$ cross section as a function of rapidity for two $p_{T}^{\mu}$ thresholds. The data sample corresponds to $4.7 \mathrm{pb}^{-1}$ and contains opposite sign muon candidates reconstructed in the muon system with a match in the central tracker.

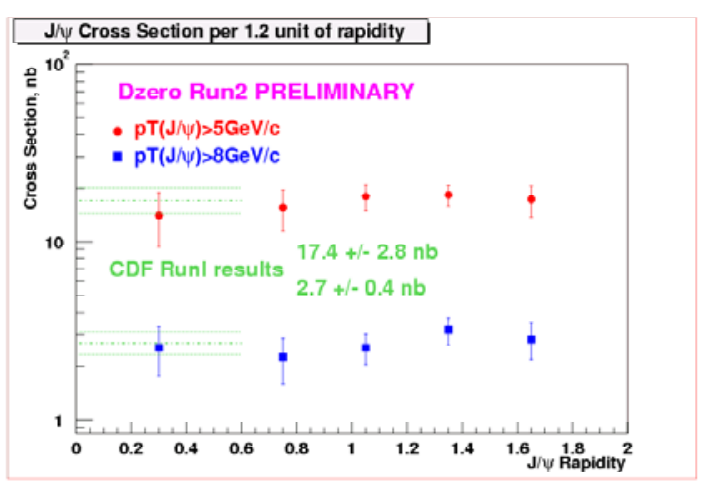

Figure 4. $J / \Psi$ cross section as a function of rapidity for two $p_{T}^{J / \Psi}$ thresholds.

The $B$ average lifetime for a $B \rightarrow J / \Psi+X$ sample, with $p_{T}^{\mu}>2 \mathrm{GeV} / \mathrm{c}$ and $p_{T}^{J / \Psi}>4 \mathrm{GeV} / \mathrm{c}$, has also been determined, giving the value $c \tau=492 \pm 37 \mu \mathrm{m}$, in agreement with the published value, $469 \mu \mathrm{m}$ [6].

\section{$5.2 \mathrm{~W} / \mathrm{Z}$}

So far, the analyses on $W$ and $Z$ have concentrated in the electron decay mode. Electrons are identified as energy clusters in the electromagnetic (EM) calorimeter, optionally associated with a track. The fraction $E_{E M} / E_{\text {total }}$ of their energy deposited (using a cone of size $\mathcal{R}=0.2$ ) in the calorimeter EM section is required to be greater than 0.90 and the shower shape must be consistent with Monte Carlo simulations of the detector. Clusters are also subject to an isolation cut, requiring that the total energy contained within $\mathcal{R}=0.4$ be less than $1.15 E_{E M}$. The selection efficiency of these cuts computed from a $Z$ sample is $0.892 \pm 0.026$.

Candidate $Z$ events are selected by requiring two EM clusters with $E_{T}>25 \mathrm{GeV}$ within $|y|<1.1$ while $W$ candidates are required to have only one EM cluster with $E_{T}>25 \mathrm{GeV}$ within $|y|<0.8$ and $\boldsymbol{E}_{T}>25 \mathrm{GeV}$. The QCD background has little effect on the $Z$ sample but is a major concern for the $W$ sample. Most of this background can be removed by requiring a track match. A $Z \rightarrow e e$ peak in the dielectron spectrum can be seen in Fig. 5 .

Using this set of cuts, preliminary $W$ and $Z$ cross section measurements were made, based on $7.52 \pm 0.75 \mathrm{pb}^{-1}$ of integrated luminosity. A complete physics and detector simulation was carried out and data corrected accordingly. The $Z$ and $W$ production cross sections times branching fractions to electrons were determined to be:

$$
\begin{aligned}
\sigma_{Z} \times B(Z \rightarrow e e)=266 & \pm 20_{\text {stat }} \pm 20_{\text {syst }} \\
& \pm 27_{\text {lumi }} \mathrm{pb} \\
\sigma_{W} \times B(W \rightarrow e e)=2.67 & \pm 0.06_{\text {stat }} \pm 0.33_{\text {syst }} \\
& \pm 0.27_{\text {lumi }} \mathrm{nb}
\end{aligned}
$$

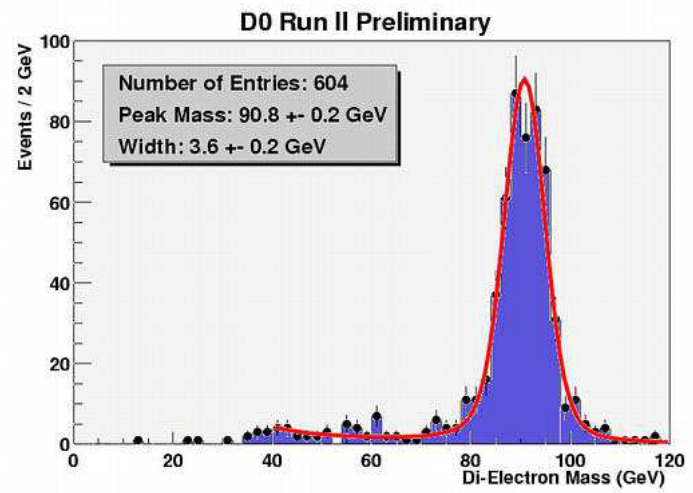

Figure 5. Dielectron mass spectrum showing the $Z$ peak.

These results are plotted in Fig. 6 along with other experimental results and theoretical predictions [7] using CTEQ4M parton distribution functions [8]. Many uncertainty sources affecting both measurements are correlated and can be eliminated, at least partially, by computing the ratio of the cross sections:

$$
R=\frac{\sigma_{W} \times B(W \rightarrow e e)}{\sigma_{Z} \times B(Z \rightarrow e e)}=10.0 \pm 0.8_{\text {stat }} \pm 1.3_{\text {syst }}
$$

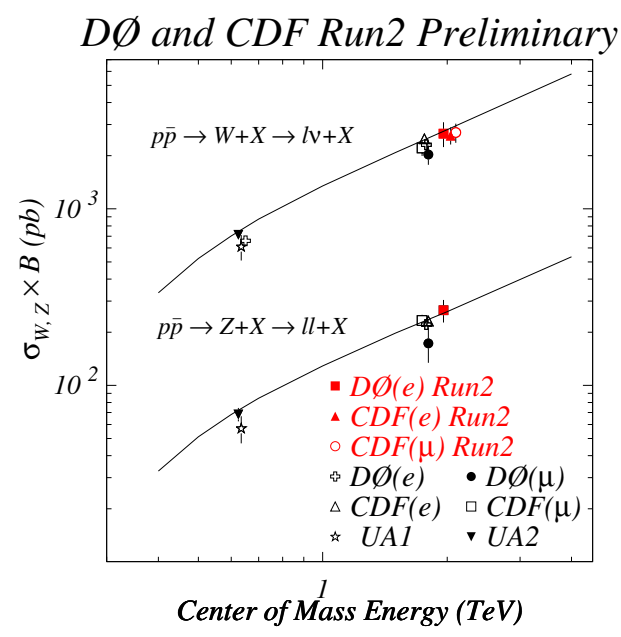

Figure 6. $W$ and $Z$ production cross sections. 
Finally, assuming $\sigma_{Z} / \sigma_{W}=0.2964 \pm 0.0047$ from theory, the Standard Model (SM) prediction $\Gamma_{W \rightarrow e \nu}=$ $0.2270 \pm 0.0011 \mathrm{GeV}$ and using $B(Z \rightarrow e e)=0.03367 \pm$ 0.00005 from LEP measurements, the $W$ total width can be extracted

$$
\Gamma_{W}=2.26 \pm 0.18_{\text {stat }} \pm 0.29_{\text {syst }} \pm 0.04_{\text {theory }} \mathrm{GeV}
$$

which is in agreement with the SM predicted value $\Gamma_{W}=$ $2.094 \pm 0.006 \mathrm{GeV}$.

\subsection{Top Quark}

The top quark production at Tevatron may occur in pairs through the strong interaction processes $\bar{q} q \rightarrow \bar{t} t$ and $g g \rightarrow$ $\bar{t} t$ or through electroweak single top processes involving a $W t b$ vertex. At $\sqrt{s}=1.8 \mathrm{TeV}$ the predicted cross sections for pair and single top production are approximately $5 \mathrm{pb}$ and $2.2 \mathrm{pb}$, and are expected to increase by nearly the same amount $(\sim 30-40 \%)$ at $\sqrt{s}=1.96 \mathrm{TeV}$.

Depending on the decay modes of the $W$ 's that come from the $t$ and/or $\bar{t}$ decays, different event topologies may arise. In top pair production, there are three possibilities: if both $W$ 's decay in leptons then a final state with two high $p_{T}$ leptons, two jets and energy imbalance $\left(\boldsymbol{E}_{T}\right)$ from the escaping neutrinos is expected to be observed; if one $W$ only decays leptonicaly then a final state with a high $p_{T}$ lepton, four jets and $\boldsymbol{E}_{T}$ is expected; if both $W$ 's underway a hadronic decay then an all-jets final state with six jets should be observed. Searches for single top production at $\mathrm{D} \varnothing$ have been restricted so far to the leptonic $W$ decay mode, leading to final states with a high $p_{T}$ lepton, $\boldsymbol{E}_{T}$ and two or three jets.

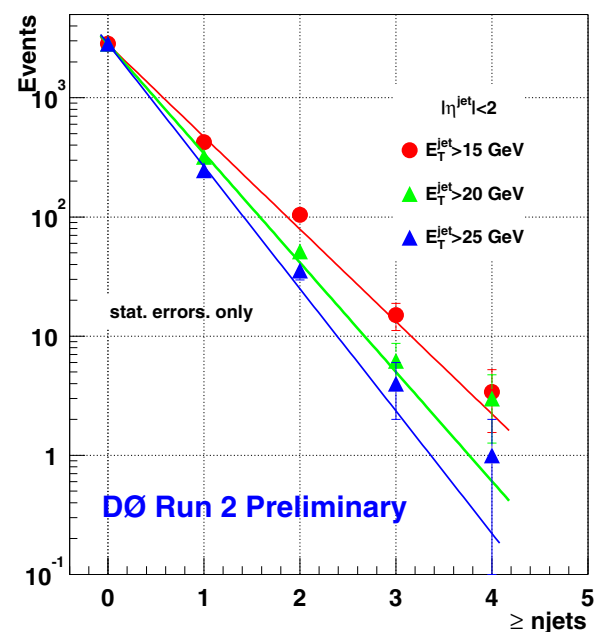

Figure 7. Inclusive jet multiplicity in background-subtracted $W \rightarrow e \nu$ events.

The amount of data collected in Run II is not yet sufficient to observe the top quark. Nevertheless, $D \varnothing$ has been studying the production of $W$ and $Z$ bosons in association with jets, as this composes the dominant background to top production. Fig. 7 shows the distribution of jet multiplicity in $W \rightarrow e \nu$ events, after subtraction of background due to events where jets were misidentified as electrons, for different jet $E_{T}$ thresholds. With increased statistics and improved $b$-jet tagging an enhancement of events with four or more jets, signaling top quark production, is expected.

\subsection{Higgs Boson}

The present data sample has no sensitivity to the production of a SM Higgs boson, with an estimated cross section of a few $\mathrm{fb}$, or even to the production of alternative models [9] Higgs, whose cross sections might be an order of magnitude higher.

A SM Higgs boson may decay to a dielectron $+\boldsymbol{E}_{T}$ final state via the decay mode $H \rightarrow W W^{(*)} \rightarrow e \nu e \nu$, but a number of other SM processes may also give two high $p_{T}$ electrons in the final state, the most important ones being $Z, \gamma^{*} \rightarrow e e$.

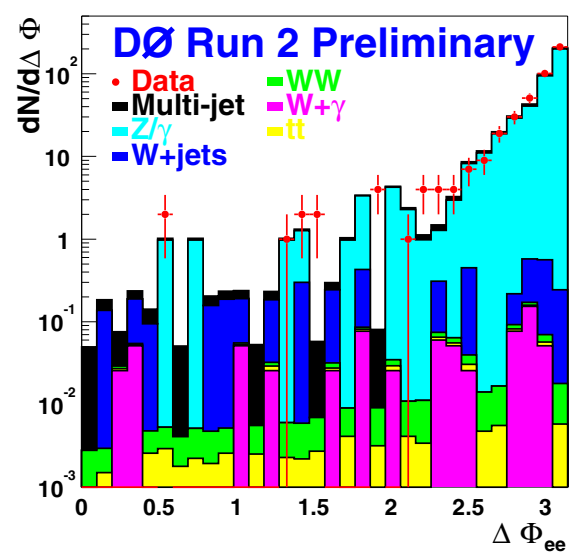

Figure 8. Data vs. Monte Carlo distributions of $\Delta \phi_{e e}$ for the dielectron $+\boldsymbol{E}_{T}$ final state.

D $\varnothing$ has studied its data in order to assess the background in final states containing this characteristic topology. The $8.8 \mathrm{pb}^{-1}$ data sample used in this study was collected using a two level single-electron trigger. Standard electron identification cuts and kinematic requirements were applied to the electron candidates in this sample. To further improve background rejection, only those events with at least one electron candidate having a match in the central tracker and with both electrons having $p_{T}>20 \mathrm{GeV} / \mathrm{c}$ were kept. After applying these cuts, the sample was dominated by $Z \rightarrow e e$ decays. This contribution was rejected by cutting on the electron pair invariant mass, $m_{e e}<78 \mathrm{GeV} / \mathrm{c}^{2}$. A few additional cuts were applied to take into account the expected topology of the Higgs events. Only one event survived all cuts.

A complete physics and detector simulation was carried out for all processes contributing to the dielectron $+\boldsymbol{E}_{T}$ final state. Then, the selection criteria were applied to all the samples. In an alternative model involving a fourth fermion generation, the expected number of signal events is 0.02 . The sum of all the background events give $0.3 \pm 1.2$ events, which agrees with one remaining event in the data sample. 
Actually the agreement holds at every stage of the analysis. The $\Delta \phi_{e e}$ distributions for data and Monte Carlo after applying kinematic and electron identification cuts are shown in Fig. 8.

\subsection{New Phenomena}

$\mathrm{D} \varnothing$ has performed searches for a variety of phenomena beyond the scope of the Standard Model: $R$-parity violating (RPV) decays of supersymmetric (SUSY) particles, gauge mediated (GMSB) SUSY, large extra dimensions, leptoquarks, squarks, gluinos, etc. Most of results are very preliminary yet.

A search for RPV SUSY decay in three leptons was performed for eee and ee $\mu$ channels and the observed number of events are in agreement with the expected physics and instrumental backgrounds. Seven events were found in the tri-electron channel, for $6 \pm 1 \mathrm{SM}$ expected background and $9 \pm 3$ estimated instrumental background. Only one ee $\mu$ candidate was found, for $0.005 \pm 0.003$ events expected from $\mathrm{SM}$ and $1.3 \pm 0.4$ from instrumental backgrounds.

RPV SUSY was also investigated in the dielectron + jets final state, which might arise through the RPV decay of a pair of SUSY particles. Due to the Majorana nature of the neutralino, the two charged leptons in the final state may be like-sign or opposite-sign with same probability. Using $9.7 \pm 1.4 \mathrm{pb}^{-1}$ of data, $\mathrm{D} \varnothing$ searched for an excess of events in any of the charge combinations but no excess was found. Data is in good agreement with SM expectation, including the jet multiplicity distribution.

D $\varnothing$ also tried to assess GMSB SUSY models by studying diphoton events with large transverse energy imbalance. Due to the low statistics available was not possible to set any meaningful limit on GMSB SUSY. On the other hand, a "model independent" limit on events with two photons $\left(m_{\gamma \gamma}\right.$ outside $80-102 \mathrm{GeV}$ ) and missing $E_{T}$ (not collinear with the leading jet) was set to be $0.9 \mathrm{pb} @ 95 \% C L$.

Recent developments in string theory suggest that the previously unreachable Plank scale $\left(\sim 10^{19} \mathrm{GeV}\right)$ might be sensed at $\mathrm{TeV}$ energy scale in the presence of extra compactified dimensions. These theories can be probed at Tevatron and $\mathrm{D} \varnothing$ has searched for large extra spatial dimensions by looking for virtual graviton effects in the fermion or boson pair production. The dimuon, dielectron and diphoton channels were examined using $10 \mathrm{pb}^{-1}$ of data and upper limits on the fundamental Plank scale $M_{S}$ were set for different theoretical formalisms $[10,11,12]$, giving values ranging from $0.73 \mathrm{GeV}$ to $1.10 \mathrm{GeV}$. These limits are based on the $\gamma \gamma$ and $e e$ channels only, since the $\mu \mu$ backgrounds are not well understood yet. Run II results on this topic are approaching Run I measurements, as can be seen by comparing $M_{S}(G R W)$ values of $0.92 \mathrm{TeV}$ (Run II) and $1.2 \mathrm{TeV}$ (Run I).

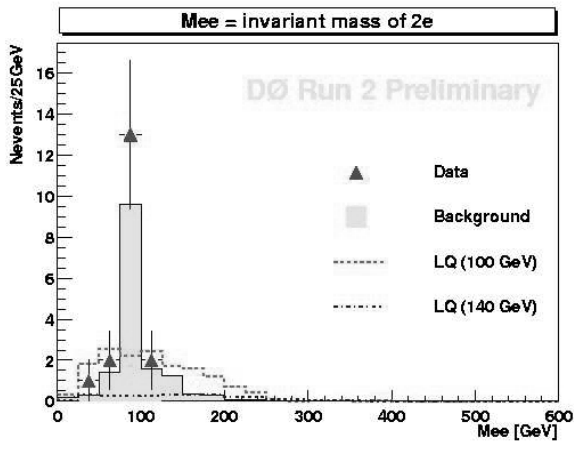

Figure 9. Dielectron invariant mass for $e e j j$ events.

First generation leptoquarks (LQ) are assumed to be produced in pairs, and searched for in the eejj final state. The dielectron invariant mass of eejj event candidates and of the estimated background are shown in Fig. 9, as well as the predicted signals for $100 \mathrm{GeV} / \mathrm{c}^{2}$ and $140 \mathrm{GeV} / \mathrm{c}^{2} \mathrm{LQ}$. From this analysis, a $95 \% C L$ lower limit of $113 \mathrm{GeV} / \mathrm{c}^{2}$ on the first generation LQ mass is derived, which is quite far yet from the final Run I data limit of $225 \mathrm{GeV} / \mathrm{c}^{2}$. However, this is consistent with the corresponding integrated luminosities of the related data samples. The current result is based on only $8 \mathrm{pb}^{-} 1$ while the Run I result was based on $115 \mathrm{pb}^{-} 1$.

\subsection{QCD}

DØ Run I jet measurements [13] provided powerful new information about the structure of the proton, which has been incorporated to recent determinations of parton distribution functions by CTEQ [14] and MRST [15] collaborations.

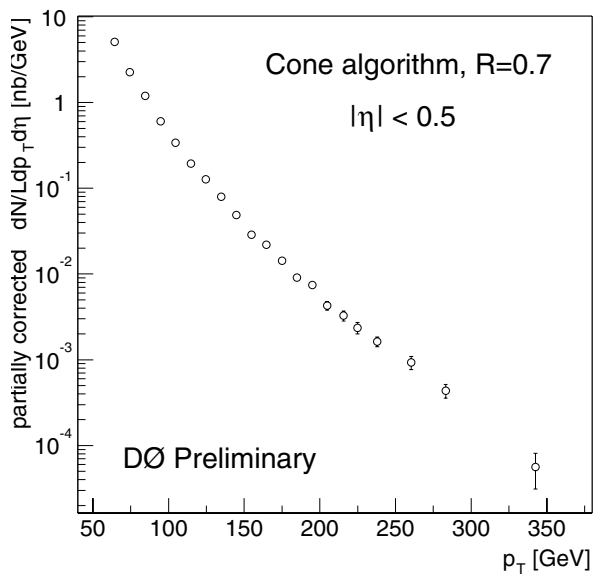

Figure 10. Inclusive jet $p_{T}$ spectrum.

In Run II, DØ plans to extend the kinematic range probed by jet measurements, especially due to the increased statistics relative to Run I and due to the increase in very 
high- $p_{T}$ jet production. Figs. 10 and 11 show the preliminary measurements of the inclusive jet production cross section and dijet mass cross section, respectively, based on $\approx 6 \mathrm{pb}^{-1}$ of data. The data $\eta$ range is limited by the fact that only central jet trigger $(|\eta<0.8|)$ was implemented during that period. An extended trigger coverage has recently become operational and new data should benefit of it.

\section{Forward Proton Detector}

The Forward Proton Detector (FPD) [16] is part of the DØ upgraded detector and comprises six stations housing Roman Pots instrumented with scintillating fiber position detectors. It is intended to provide trigger and tracking capabilities for detecting near beam protons and/or antiprotons scattered in elastic or diffractive collisions.

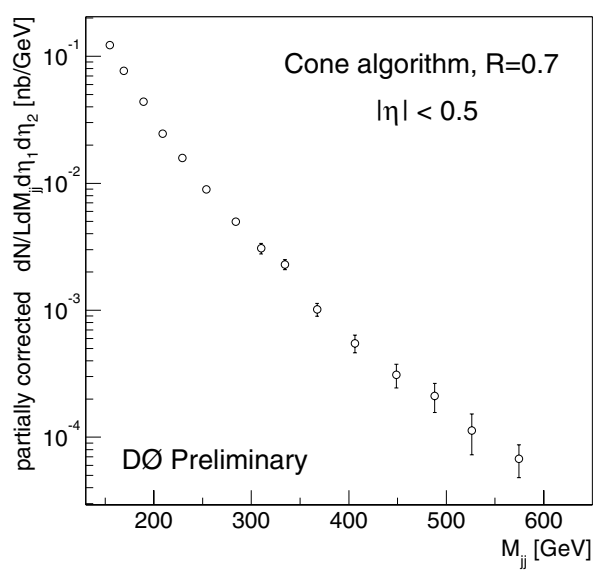

Figure 11. Dijet invariant mass spectrum.

The stations are located along the beam pipe at both sides of the $\mathrm{D} \emptyset$ : $\mathrm{P} 1$ and $\mathrm{P} 2$ on the proton side (side to where protons are moving) and $\mathrm{A} 1, \mathrm{~A} 2, \mathrm{D} 1$ and D2 on the antiproton side. A1, A2, P1 and P2 have four instrumented arms, two horizontal (IN and OUT) and two vertical (UP and DOWN), are placed before the Tevatron closest dipole magnets and are called quadrupole stations. D1 and D2 are located after the dipole magnet, have only one arm (IN) and are called dipole stations. Distances from the center of the DØ vary from $23 \mathrm{~m}$ (A1 and P1) to $59 \mathrm{~m}$ (D2). Same arms in consecutive stations (e.g. P1UP and P2UP) form a FPD spectrometer. Tracks are reconstructed by spectrometer.

Placed inside the station arms are the position detectors, made up of six planes of scintillating fibers in three views: $\mathrm{U}, \mathrm{U}^{\prime}, \mathrm{X}, \mathrm{X}^{\prime}, \mathrm{V}, \mathrm{V}^{\prime}$. X planes are perpendicular to the bottom edge of the Roman pot and have 16 fibers. $\mathrm{U}$ and $\mathrm{V}$ planes are rotated by $\mp 45^{\circ}$ with respect to $\mathrm{X}$ and have 20 fibers. The fiber width is $0.8 \mathrm{~mm}$ and adjacent fibers are separated by $0.27 \mathrm{~mm}$. Primed and unprimed planes are offset by $2 / 3$ of a fiber. A thin scintillator piece for trigger purposes complete a position detector. Fig. 12 shows a picture of a fully instrumented dipole detector installed in the beam line.

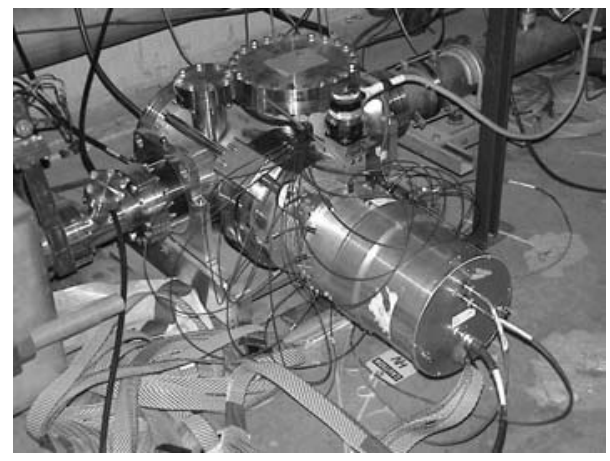

Figure 12. FPD dipole detector installed at Tevatron beam line.

With the addition of the FPD, a broad program in hard and soft diffraction and topics on elastic scattering is planned to be carried out in Run II: $p \bar{p}$ total cross section and $t$-dependence of elastic scattering; inclusive single diffraction and double pomeron studies; diffractive production of high- $p_{T}$ and high-mass states (jets, heavy quarks, $\gamma$, $Z, W$, Higgs); search for Centauros, glueballs and other exotic states.

Detector installation and commissioning has not been finished yet, and due to technical and founding reasons it has been divided in two phases. PHASE I should be essentially finished by October 2002, with 10 out of the 18 position detectors installed and ready to be commissioned. Presently, 9 detectors are already installed. PHASE II should take longer and still depends upon some fund availability. With PHASE I, we aim to have all vertical pots plus the dipoles instrumented.

The FPD is not fully integrated yet to the rest of DØ experiment and has been operating through a stand alone data acquisition (DAQ) system, which is capable of reading out only two detectors at the same time. Installation of $\mathrm{D} \emptyset$ standard DAQ electronics is underway. The dipole spectrometer has already been connected and is being commissioned. Analog front end boards (AFE) read detector channels out, digitize them and send them through to digital front end boards (DFE), where trigger logic is implemented into field programmable gate array (FPGA) elements. DFE decisions are then sent to a FPD trigger manager (TM) board which by its turn send FPD trigger information to the $\mathrm{D} \varnothing$ trigger framework.

Data collected through the stand alone DAQ system is currently being analyzed. Detectors channels status are being assessed by looking at ADC distributions. Possibility of using timing information from trigger scintillators for vetoing the beam halos background is under investigation. Studies of position correlation between the pair of detectors that make up a spectrometer are helping to align the system. Preliminary results show a position resolution of $110 \mu \mathrm{m}$ for dipoles that approaches theoretical limit of $80 \mu \mathrm{m}$. 


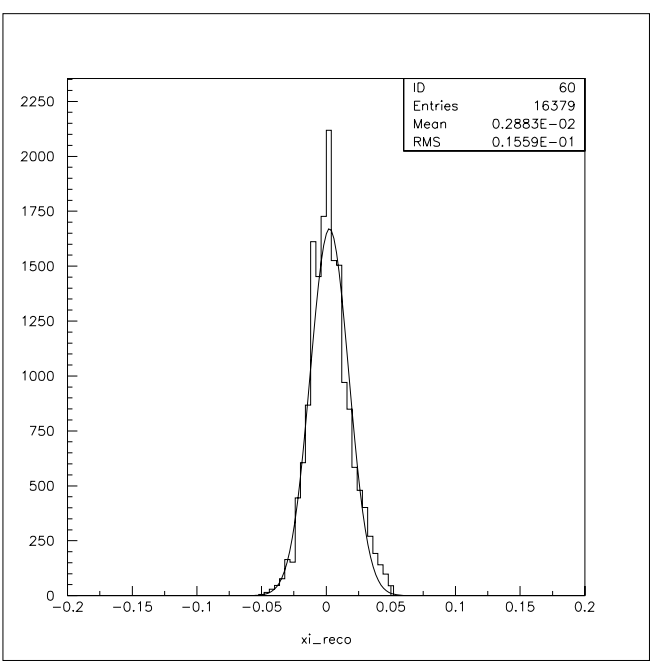

Figure 13. $\xi$ distribution of elastic candidates.

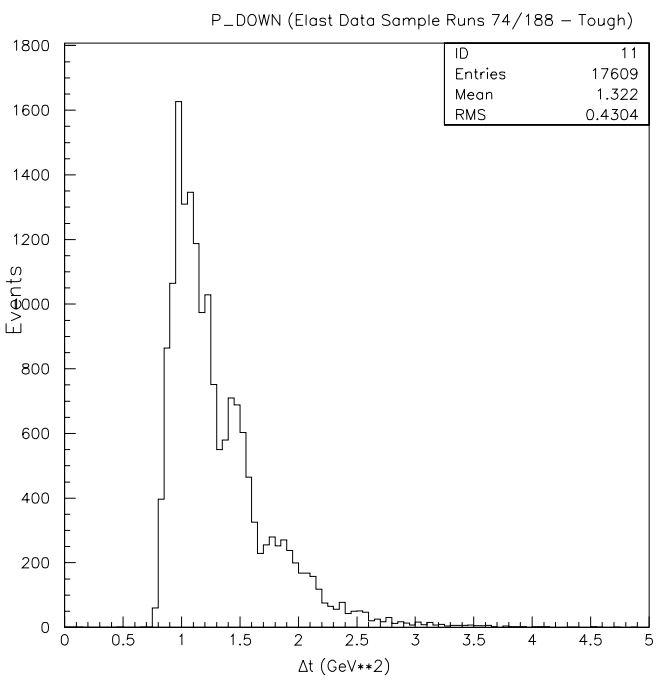

Figure 14. $|t|$ distribution of elastic candidates.

The first very preliminary physics distributions on $\xi$ and $t$ from elastic data have been produced and are shown here in Figs. 13 and 14. Their shapes are consistent with expectations but not much information can be extracted yet from these distributions. More statistics and further detector studies are needed.

\section{Summary}

DØ has undergone a major upgrade from Run I to Run II and is almost full operational now. It has started to produce physics results and our understanding of the detector subsystems and data is rapidly improving.
Tevatron performance also is improving with a significant increase in delivered luminosity over the last few months. Now, it is time to accumulate more data and refine the preliminary results presented in this article.

FPD also has made a great progress. We are finishing the PHASE I detectors installation and are integrating FPD to the DØ DAQ system.

Through a stand alone DAQ system, we were able to record a good amount of FPD data, especially elastic events. Some very preliminary physics distributions have been derived from this data.

More important, FPD is well on the way to completion and full integration to $\mathrm{D} \emptyset$, and the whole experiment is ready to a very exciting Run II.

I warmly thank all my Collaboration colleagues, whom have, through their hard work, made this presentation possible.

\section{References}

[1] DØ web site: http://www-d0.fnal.gov/.

[2] Physics Highlights from the DØ Experiment $1992 \sim 1999$, not published, available from Fermilab.

[3] DØ Collaboration, S. Abachi et al., Nucl. Inst. and Meth. A338, 185 (1994).

[4] DØ Collaboration, The DØ Upgrade: The Detector and its Physics, Fermilab Pub-96/357-E (1996).

[5] $y$ is the particle rapidity defined as $y=\frac{1}{2} \ln \frac{E+p_{z}}{E-p_{z}}$. Rapidity and the pseudo-rapidity $\eta \equiv-\ln [\tan (\theta / 2)]$, where $\theta$ is the polar angle, will be used interchangeably.

[6] Particle Data Group, Phys. Rev. D66, 1 (2002).

[7] R. Hamberg, W.L. van Neerven, and T. Matsuura, Nucl. Phys. B359, 343 (1991); W.L. van Neerven, E.B. Zijlstra, Nucl. Phys. B382, 11 (1992).

[8] CTEQ Collaboration, H.L. Lai et al., Phys. Rev. D55, 1280 (1997).

[9] L. Brücher, R. Santos, Eur. Phys. J. C 12, 97 (2000); E. Arik et al., hep-ph/0109037.

[10] G. Giudice, R. Rattazzi, and J. Wells, Nucl. Phys. B544, 3 (1999).

[11] T. Han, J. Lykken, and R. Zhang, Phys. Rev. D59, 105006 (1999).

[12] J. Hewett, Phys. Rev. Lett. 82, 4765 (1999).

[13] DØ Collaboration, B. Abbott et al., Phys. Rev. Lett. 86, 1707 (2001).

[14] J. Pumplin, D.R. Stump, J. Huston, H.L. Lai, P. Nadolsky, and W.K. Tung, JHEP. 0207, 012 (2002).

[15] A.D. Martin, R.G. Roberts, W.J. Stirling, and R.S. Thorne, Eur. Phys. J. C 23, 73 (2002).

[16] A. Brandt et al., A Forward Proton Detector at DØ, Fermilab Pub-97/377-E (1997). 